\title{
INVERSIÓN EXTRANJERA DIRECTA, EXPORTACIONES Y CRECIMIENTO ECONÓMICO EN AMÉRICA LATINA
}

\section{FOREIGN DIRECT INVESTMENT, EXPORTS AND ECONOMIC GROWTH IN LATIN AMERICA}

\author{
Galo Acosta Palomeque \\ 〈gracosta@espe.edu.ec〉 \\ Henry Pazmiño Arroyo \\ 〈henryh.pazmiño@ute.edu.ec〉 \\ Nelson Cerda Prado \\ 〈ncerda@ute.edu.ec $\rangle$
}

\section{Resumen}

Del análisis de la relación de la Inversión Extranjera Directa (IED) y de las exportaciones con el crecimiento económico de 16 países de Latinoamérica durante el año 2015. En este sentido, se desarrolló un modelo de regresión lineal múltiple, utilizando una serie transversal de datos para el análisis de los países. Los resultados identificados permiten concluir que en los países latinoamericanos analizados, la IED y las exportaciones impulsan el crecimiento económico, lo cual es un dato relevante para los organismos que hacen políticas públicas y es concordante con la mayoría de literatura al respecto. Si bien la variable volumen negociado en el mercado de valores fue descartada por tener una alta correlación con la IED y por ser la menos correlacionada con la variable dependiente, a los diferentes países les corresponde incentivar a este mercado que constituye para las empresas una importante fuente de financiamiento.

Palabras clave: crecimiento económico, exportaciones, inversión extranjera directa, Latinoamérica, mercado de valores.

\section{Abstract}

This paper analyzes the relationship between Foreign Direct Investment and Exports with the economic growth of 16 Latin American countries during the year 2015. In this sense, a multiple linear regression model was developed using a cross-sectional series of data for the analysis of the countries. The results show that in the Latin American countries studied, Foreign Direct Investment and Exports promote economic growth, which is an important fact for the organisms that make public policies and is in agreement with the majority of literature on the matter. Although the variable volume traded in the stock market was discarded because it has a high correlation with Foreign Direct Investment and because it is the least correlated with the dependent variable, the different countries have the incentive to this market that constitutes for the companies a important source of funding.

Keywords: Economic growth, exports, Foreign Direct Investment, Latin America, stock market.

\section{INTRODUCCIÓN}

La relación teórica entre mercado de valores y crecimiento económico se evidencia en los países que poseen 
mercados de capitales más eficientes y profundos, y que están en condiciones de lograr tasas de crecimiento más altas, en contraposición los países con menor desarrollo económico se caracterizan por tener mercados de valores pequeños. En este sentido es importante identificar la relación existente entre el volumen negociado en los mercados de valores latinoamericanos y el crecimiento económico en estos países.

Al comercio internacional se le considera como uno de los factores que mayor crecimiento económico genera a las economías, ya que a través del intercambio de bienes y servicios los países son capaces de adquirir en el extranjero los productos que les resulta costosos producir internamente. El comercio internacional permite a los países mejorar sus técnicas de producción y por ende su competitividad, que se reflejará en una mayor eficiencia y calidad de los productos elaborados para consumo interno o para exportación.

Smith (1776) pudo observar que las limitaciones que tenían los mercados internos de los países para aumentar la acumulación de capital. Por ello, visualizó en el comercio exterior los potenciales beneficios para las naciones que lo practican. En este sentido, tanto las importaciones como las exportaciones son medios que se deben utilizar para aumentar la riqueza y bienestar de la sociedad. Caso contrario, los países con economías abiertas con dificultades en la balanza de pagos y que limitan sus importaciones a través de barreras arancelarias y no arancelarias experimentarán una contracción de su crecimiento económico.

Durante las décadas de 1950 y 1960 , varios autores consideraban que la inversión extranjera afectaba de forma negativa el crecimiento económico de los países receptores. Posteriormente, gracias al crecimiento logrado por muchos países, a mediados de 1970 esta discusión se modificó dando lugar a las nuevas teorías de crecimiento.

La muestra abarca datos del año 2015 de 16 países latinoamericanos. La selección de los mismos corresponde al interés de estudiar el impacto de las exportaciones, Inversión Extranjera Directa y del Mercado de Valores en el crecimiento económico de las economías latinoamericanas. Para ello, se aplica un Modelo Lineal de Regresión Múltiple para el análisis de una serie transversal de datos

$\begin{array}{cccc}\text { Los } & \text { hallazgos } & \text { de } & \text { la } \\ \text { investigación } & \text { concuerdan } & \text { con } & \text { lo }\end{array}$ indicado en gran parte de la literatura actual. Esto es, la inversión extranjera directa y las exportaciones tienen un efecto positivo y significativo en el crecimiento económico.

\section{MARCO TEÓRICO}

\section{Mercado de valores y crecimiento económico}

La relación teórica entre mercado de valores y crecimiento económico se evidencia en los países que poseen mercados de capitales más eficientes y profundos quienes están en condiciones de lograr tasas de crecimiento más altas, dado que las bolsas de valores facilitan el financiamiento de la inversión, mejoran la asignación de recursos, admiten una administración más eficiente de los riesgos y permiten corrientes más estables de consumo e inversión (Marshall \& Walker, 2000)

(Rueda, 2005) plantea la importancia del mercado de valores desde cuatro enfoques principales:

1. El mercado es el sitio donde el Banco Central lleva a cabo las operaciones de mercado abierto, uno de los instrumentos más importantes de la política monetaria y crediticio. 
2. La bolsa concentra recursos que representan una parte importante del ahorro interno del país.

3. Es la puerta de entrada de los flujos de inversión directa del exterior.

4. El mercado de valores impacta en el desarrollo de la economía.

Al respecto, solo aquellos países que presentan mayores índices de formación bruta de capital fijo, han conseguido un crecimiento económico sostenido. Los mercados de valores de renta fija y, principalmente, el de renta variable, inciden en el crecimiento económico.

Hicks (1969) señala que las mejoras en el sistema financiero inglés y de su mercado de capitales fueron las principales causas de la Revolución industrial en Inglaterra. Demirguc-Kunt \& Levine (1993) aseguran que un creciente número de investigaciones, tanto teóricas como empíricas, tienden a inclinar incluso a los escépticos hacia la creencia de que el desarrollo de los mercados financieros es un determinante del crecimiento económico, más que un acompañamiento o una respuesta pasiva a este crecimiento.

El estudio de Levine \& Zervos (1998) muestra que uno de los factores de mayor relevancia para el crecimiento a largo plazo, lo constituye precisamente el mercado de valores. En este estudio se relacionan la liquidez del mercado de valores con el crecimiento económico nacional, las tasas de acumulación de capital y los coeficientes de cambio tecnológico.

El desarrollo económico sostenido resulta no solo de la combinación de la actividad empresarial y de la inversión en capital tangible e intangible, sino también de la intermediación financiera que realiza el mercado de capitales.

En general, explica Arbeláez (2004), existen cuatro grandes corrientes teóricas que establecen una relación entre el sector financiero $y$ el crecimiento económico, ellas son:

1. En primer lugar, la visión basada en el papel de los bancos, según la cual la movilización de recursos, la identificación de buenos proyectos y la administración del riesgo por parte de los bancos son factores que se anteponen al mercado de valores.

2. La visión basada en el mercado de valores que resalta el papel de los mercados en promover el éxito del sector real, en la medida que facilita la diversificación y los instrumentos para la administración de los riesgos.

3. La visión de ley y finanzas, la cual rechaza la hipótesis de que exista una relación entre la estructura financiera $\mathrm{y}$ el crecimiento económico $\mathrm{y}$, más bien, insiste en la relevancia del sistema legal y su vínculo positivo con el crecimiento.

4. Finalmente, la visión de los servicios financieros argumenta que el sistema financiero provee servicios cruciales para el crecimiento económico, de manera independiente del mercado de origen.

\section{Importaciones $\quad y \quad$ crecimiento económico}

El crecimiento económico es una condición necesaria para el desarrollo económico de los países. Este crecimiento se puede dar de muchas formas y una de ellas es a través del sector externo de la economía.

$\mathrm{Al}$ comercio internacional se le considera como uno de los factores que mayor crecimiento económico genera a las economías, ya que a través del intercambio de bienes y servicios los países son capaces de adquirir en el 
extranjero los productos que resulta costoso producir internamente. Adicionalmente, el comercio internacional es una fuente de ingreso de tecnología, ya sea a través de la importación de maquinaria y equipos o por el ingreso de conocimientos, permitiendo a los países receptores mejorar sus técnicas de producción, y por ende su competitividad que se reflejará en una mayor eficiencia y calidad de los productos elaborados para consumo interno o para exportación.

Smith (1776) pudo observar las limitaciones que tenían los mercados internos de los países para aumentar la formación de capital. Por ello, visualizó en el comercio exterior los potenciales beneficios para las naciones que lo practiquen. En este sentido, tanto las importaciones como las exportaciones son medios que se deben utilizar para incrementar el nivel de vida de los habitantes de un país.

El modelo de Thirwall (2003) supone que un país no puede recurrir a las instituciones financieras $\mathrm{u}$ a otros países para financiar indefinidamente su déficit en la balanza de pagos. A largo plazo la tasa de incremento de la demanda agregada estará condicionada por la disponibilidad de divisas extranjeras. El autor en la evidencia empírica concluye que a largo plazo el crecimiento económico estuvo influenciado, particularmente, por la elasticidad ingreso de las importaciones y la tasa de incremento de las exportaciones y que los niveles de términos de intercambio influyeron en el crecimiento

De su lado, la Comisión Económica para América Latina y el Caribe (CEPAL, 2004) presenta argumentos respaldando el papel que juegan las exportaciones en el crecimiento económico de los países. Se enfatiza que las exportaciones generan divisas a un país; las cuales son fundamentales para el crecimiento económico, ya que por medio de las importaciones de insumos intermedios $y$ bienes de capital que no se producen internamente se logra fortalecer la actividad económica.

\section{Inversión extranjera y crecimiento económico}

Una de las características más importantes de la globalización en la actualidad es la IED. La inversión extranjera es una categoría de inversión internacional que tiene como objetivo obtener una participación duradera en una empresa residente en otra economía. La inversión directa comprende tanto la transacción inicial entre inversor y empresa como también todas las transacciones que tengan lugar, posteriormente, entre ello. La teoría del crecimiento endógeno estudia la inversión extranjera como uno de los determinantes del crecimiento económico de los países.

A través de la inversión extranjera directa que realizan las grandes corporaciones multinacionales se posibilita la transferencia de tecnología desde los países avanzados hacia los países menos desarrollados, permitiendo a estos últimos obtener mayores tasas de crecimiento económico.

Durante las décadas de 1950 y 1960, varios autores consideraban que la inversión extranjera afectaba de forma negativa el crecimiento económico de los países receptores. Posteriormente, gracias al crecimiento logrado por muchos países, a mediados de 1970 esta discusión se modificó dando lugar a las nuevas teorías de crecimiento. Estas teorías permiten 
analizar desde otra perspectiva la relación existente entre IED y crecimiento económico.

En el contexto de las nuevas teorías de crecimiento, la IED afecta el crecimiento económico de un país de manera endógena. Blomstrom y $\mathrm{Ye}$ Wang (1989) identificaron a la variable inversión extranjera directa para explicar el crecimiento económico de los países menos desarrollados, donde la innovación tecnológica juega un rol esencial. Esto resulta factible si se generan rendimientos crecientes en la producción, produciendo crecimiento económico a largo plazo. Desde la perspectiva de las finanzas internacionales se sugiere que la IED tiene la capacidad de impactar positivamente en la economía receptora, por ejemplo, a través de la transferencia de tecnología (Kozikowsky, 2013).

Desde el planteamiento de la teoría de la organización industrial se considera que John Dunning ha realizado las mayores contribuciones, que han sido enriquecidos por otros economistas como Caves, Heymer, Vernon, entre otros. Se estudian los factores de localización de las empresas multinacionales, las ventajas que llevan a las organizaciones a decidirse a operar en países diferentes al de origen.

La capacidad y disposición para que las empresas operen en su país o en el extranjero, según Dunning y Lundan (2008), se encuentra en función de los dos siguientes elementos:

1. La capacidad de poseer o adquirir ciertos activos no disponibles en términos ventajosos, a esta situación se denomina ventaja de propiedad específica $\langle\mathrm{O}\rangle$.

2. El disponer de activos antes señalados, puede estar condicionada a una ubicación geográfica en particular, se denomina ventaja de localización $\langle\mathrm{L}\rangle$.

Según Rivas \& Puebla (2016) hoy en día en los trabajos de investigación más importantes, mediante el uso de la estadística y la econometría, se busca demostrar los beneficios o no de la inversión extranjera directa en el crecimiento económico de las naciones. Una investigación sobre la IED en México realizada por Dussel \& Galindo (2007), determina que es una variable importante que fomenta el crecimiento económico debido a que inyecta nuevos recursos que permiten equilibrar la balanza de pagos y motiva la transformación industrial al producir efectos que se traducen en aumentos de la productividad, del valor agregado $\mathrm{y}$ de las exportaciones.

\section{METODOLOGÍA}

Se utilizó la técnica de revisión de registros, la metodología de indagación documental, descriptiva, inductiva y experimental dado que el procedimiento implica la búsqueda, organización, sistematización y análisis de documentación sobre el crecimiento económico y su relación con el mercado de valores, inversión extranjera directa y las exportaciones.

Las unidades de análisis fueron todos los documentos sobre los temas encontrados en las bases de datos Scielo, Prosquest, Redalyc, Dialnet, Universidades y en Google Académico. Como criterios de búsqueda, se incluyeron los siguientes descriptores: "crecimiento económico", "inversión extranjera directa", "exportaciones", "regresión múltiple". Estos descriptores fueron combinados de diferentes formas para ampliar los criterios de búsqueda.

Al realizar la búsqueda se seleccionó 10 libros físicos y digitales 
de autores clásicos e informes, como también 9 artículos de bases de datos indexadas, y se revisaron sistemáticamente estos documentos relacionados con la inteligencia emocional y la gestión de las emociones en las organizaciones.

Para la organización de los documentos se creó una base de datos con los siguientes campos: título del libro o artículo, autor, año, revista, objetivos, tipo de investigación, método, resultados y núcleo temático. Una vez organizada la información, se agruparon los documentos por núcleos temáticos. Posteriormente, se realizó el análisis de cada uno de los núcleos temáticos. Finalmente, se efectuó un análisis global

mediante el cual se identificaron las convergencias y divergencias de los modelos de inteligencia emocional y se formularon ciertas conclusiones.

\section{Muestra}

- $\quad$ Ied $=$ Inversión Extrajera Directa en
La muestra abarca datos del año 2015 para los siguientes países: Bolivia, Brasil, Chile, Costa Rica, Ecuador, El Salvador, Guatemala, Honduras, México, Nicaragua, Panamá, Paraguay, Perú, República Dominicana y Uruguay. La selección de los mismos corresponde al interés de estudiar el impacto de las exportaciones, inversión extranjera directa y del mercado de valores en el crecimiento económico de las economías latinoamericanas. Para ello, se aplica un Modelo de regresión lineal múltiple para el análisis de una serie transversal de datos

Las variables explicativas consideradas en la investigación como factores del crecimiento económico surgen de la literatura mencionada con anterioridad y son las siguientes:

- $\mathrm{Y}_{\mathrm{it}}=$ Producto Interno Bruto en millones de dólares corrientes

- Expor $_{i t}=$ Exportaciones en millones de dólares corrientes millones de dólares corrientes

\begin{tabular}{|l|r|r|r|r|r|r|r|r|}
\hline PAÍS & \multicolumn{1}{|c|}{ PIB } & \multicolumn{1}{c|}{ EXPOR } & \multicolumn{1}{c|}{ IED } & \multicolumn{1}{c|}{ MV } & \multicolumn{1}{c|}{ InPIB } & \multicolumn{1}{c|}{ InEXPOR } & \multicolumn{1}{c|}{ InIED } & \multicolumn{1}{l|}{ InMV } \\
\hline Argentina & 632841.4 & 56752.0 & 11103.5 & 80887.2 & 13.4 & 11.0 & 9.3 & 11.3 \\
\hline Bolivia & 32997.7 & 8723.0 & 495.4 & 11105.0 & 10.4 & 9.1 & 6.2 & 9.3 \\
\hline Brasil & 1774722.2 & 191134.0 & 61576.2 & 487560.0 & 14.4 & 12.2 & 11.0 & 13.1 \\
\hline Chile & 240796.4 & 63362.0 & 4663.3 & 20730.0 & 12.4 & 11.1 & 8.5 & 9.9 \\
\hline Costa Rica & 54150.0 & 9666.0 & 2541.9 & 54076.0 & 10.9 & 9.2 & 7.8 & 10.9 \\
\hline Ecuador & 100176.8 & 18366.0 & 1321.4 & 7153.1 & 11.5 & 9.8 & 7.2 & 8.9 \\
\hline El Salvador & 25850.2 & 5520.0 & 428.8 & 3816.0 & 10.2 & 8.6 & 6.1 & 8.3 \\
\hline Guatemala & 63794.2 & 10726.0 & 1337.5 & 100209.0 & 11.1 & 9.3 & 7.2 & 11.5 \\
\hline Honduras & 20175.6 & 3920.0 & 1112.8 & 8624.0 & 9.9 & 8.3 & 7.0 & 9.1 \\
\hline México & 1148059.8 & 380772.0 & 22126.6 & 110562.0 & 14.0 & 12.9 & 10.0 & 11.6 \\
\hline Nicaragua & 12692.5 & 2419.0 & 784.5 & 1207.0 & 9.5 & 7.8 & 6.7 & 7.1 \\
\hline Panamá & 52132.3 & 696.0 & 4586.0 & 5247.0 & 10.9 & 6.6 & 8.4 & 8.6 \\
\hline Paraguay & 27093.9 & 8357.0 & 260.3 & 480.0 & 10.2 & 9.0 & 5.6 & 6.2 \\
\hline Perú & 189209.8 & 33247.0 & 7690.4 & 3516.2 & 12.2 & 10.4 & 9.0 & 8.2 \\
\hline $\begin{array}{l}\text { República } \\
\text { Dominicana }\end{array}$ & 68102.6 & 9526.0 & 2199.1 & 4301.0 & 11.1 & 9.2 & 7.7 & 8.4 \\
\hline Uruguay & 53442.4 & 7679.0 & 1292.6 & 1229.0 & 10.9 & 9.0 & 7.2 & 7.1 \\
\hline
\end{tabular}


- $\mathrm{Mv}_{\mathrm{it}}=$ Volumen negociado en el Mercado de valores

El subíndice 〈i〉 representa iésimo país, mientras que el subíndice 〈t〉, el momento del evento. La CEPAL es la fuente de información para todas las variables, con excepción de MV, obtenida en los sitios web de las respectivas bolsas de valores

\section{El modelo}

El modelo desarrollado en este trabajo tiene como objetivo medir el impacto de las exportaciones, de la inversión extranjera y del mercado de valores en el crecimiento económico de los países latinoamericanos. La elección del Modelo lineal de regresión múltiple (MRLM) obedece a que reconoce la relación que existe entre una variable endógena $\langle\mathrm{Y}\rangle \mathrm{y}$ un grupo de variables exógenas 〈Xi〉 para determinar el comportamiento de las variables explicativas para realizar estimaciones de la variable endógena

La expresión general del Modelo de Regresión Lineal Múltiple, adopta la siguiente forma:

$$
\begin{aligned}
& \mathrm{Yi}=\beta 0+\beta 1 \mathrm{xi} 1+\beta 2 \mathrm{xi} 2+\ldots \\
& +\beta \mathrm{KxiK}+\varepsilon \mathrm{i}, \mathrm{i}=1, \ldots, \mathrm{n},
\end{aligned}
$$

La ecuación estimada se expresa, entonces como:

$$
\hat{y}={ }^{\wedge} \beta 0+{ }^{\wedge} \beta 1 \mathrm{x} 1+\ldots+{ }^{\wedge} \beta \mathrm{KxK} .
$$

El método de estimación a emplear es el de Mínimos Cuadrados Ordinarios (MCO), que según Álvarez, Barraza \& Legato (2009) tiene el inconveniente de que no considera el efecto individual no observable, el cual no debería estar correlacionado con las otras variables explicativas, situación que no se cumple.
En la tabla 1 se muestran los valores de las variables correspondientes al año 2016, medidas en millones de dólares corrientes y como logaritmos naturales.

\section{Tabla 1}

\section{COMPONENTES DEL MODELO} EN MILLONES DE USD Y EN

\section{LOGARITMOS}

Fuente. Obtenido de CEPAL (2016) y sitios web de las bolsas de valores latinoamericanas.

En el presente estudio, se utilizó el software SPPS para estimar los parámetros del modelo de regresión lineal múltiple.

\section{RESULTADOS Y DISCUSIÓN}

Al correr el modelo en el paquete SPSS 24 , se obtienen los siguientes resultados en la tabla 2:

Tabla 2. Coeficientes

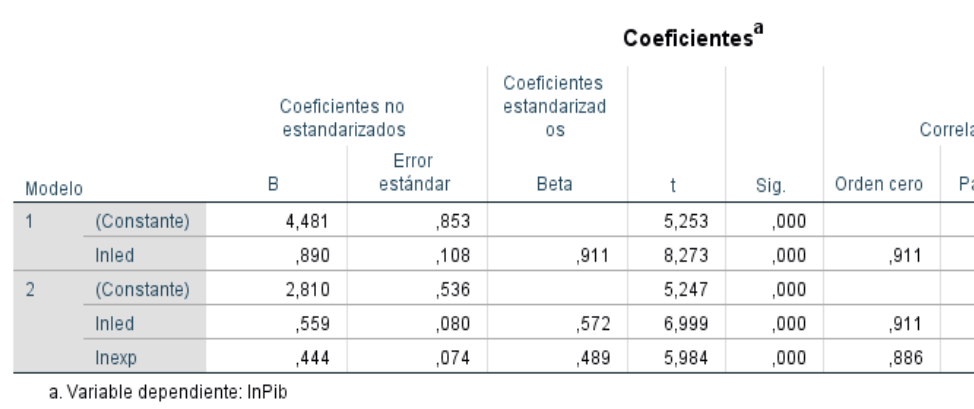

\section{MODELO PROPUESTO}

$$
\ln (\mathrm{PIB})=2.81+0.559 \ln (\mathrm{IED})+0.444 \ln (\mathrm{EXP})
$$

\section{Prueba general del modelo para todos los coeficientes juntos}

$$
\begin{aligned}
& \text { Ho: } \beta 0=\beta 1=\beta 2=0 \\
& \text { Нa: } \beta 0 \neq \beta 1 \neq \beta 2 \neq 0
\end{aligned}
$$

Se acepta la hipótesis alternativa, esto implica que el modelo es bueno para la población 
Prueba de hipótesis para cada coeficiente del modelo

Ho: $\beta 0=0$

Ho: $=\beta 2=0$

Ho: $\beta 1=0$

Ha: $\beta 0 \neq 0$

$\mathrm{Ha}:=\beta 2 \neq 0$

Ha: $\beta 1 \neq 0$

Se rechaza las hipótesis nulas, y se acepta las hipótesis alternativas, ya que los $\beta$ estimados son significativamente diferentes de cero, los niveles de significancia 0,000 son menores que 0.05. Los estadísticos $\mathrm{t}$ student son mayores de 2, cayendo en la zona de rechazo de las hipótesis nulas.

\section{Diagnóstico de colinealidad}

El factor de inflación de la varianza (VIF) indica que se cumple el supuesto de no multicolinealidad entre las variables ya que es menor a 10 en todos los casos.
Elaboración propia.

En la matriz de correlación se observa que existe una fuerte correlación entre la variable dependiente $\ln P I B$ y las variables independientes (lnEXP, $\ln I E D$ y $\ln \mathrm{MV}$ ). Asimismo, se observa que la variable lnMV sale del modelo, debido a que es la que menos se correlaciona con la variable independiente, y por presentar una alta correlación con lnIED.

Tabla 4: Resumen del modelo

\begin{tabular}{|c|c|c|c|c|c|}
\hline & & $\operatorname{lnPib}$ & Lnexp & $\operatorname{lnIed}$ & $\operatorname{lnMV}$ \\
\hline \multirow[t]{3}{*}{ LnPib } & $\begin{array}{l}\text { Correlación de } \\
\text { Pearson }\end{array}$ & 1 & $.886^{* *}$ & $0.911^{* *}$ & $0.736^{* * *}$ \\
\hline & Sig. (bilateral) & & .000 & 0.000 & 0.001 \\
\hline & $\mathrm{N}$ & 16 & 16 & 16 & 16 \\
\hline \multirow[t]{3}{*}{ Lnexp } & $\begin{array}{l}\text { Correlación de } \\
\text { Pearson }\end{array}$ & $0.886^{* *}$ & 1 & $0.693^{* *}$ & $0.644^{* *}$ \\
\hline & Sig. (bilateral) & 0.000 & & 0.003 & 0.007 \\
\hline & $\mathrm{N}$ & 16 & 16 & 16 & 16 \\
\hline \multirow[t]{3}{*}{ LnIed } & $\begin{array}{l}\text { Correlación de } \\
\text { Pearson }\end{array}$ & $0.911^{* *}$ & $0.693^{* *}$ & 1 & $0.724^{* *}$ \\
\hline & Sig. (bilateral) & 0.000 & 0.003 & & 0.002 \\
\hline & $\mathrm{N}$ & 16 & 16 & 16 & 16 \\
\hline \multirow[t]{3}{*}{ LnMV } & $\begin{array}{l}\text { Correlación de } \\
\text { Pearson }\end{array}$ & $0.736^{* *}$ & $0.644^{* *}$ & $0.724^{* *}$ & 1 \\
\hline & Sig. (bilateral) & 0.001 & 0.007 & 0.002 & \\
\hline & $\mathrm{N}$ & 16 & 16 & 16 & 16 \\
\hline
\end{tabular}

**. La correlación es significativa en el nivel 0,01 (bilateral).

\section{Matriz de correlaciones}

Tabla 3. Correlaciones 


\begin{tabular}{|c|c|c|c|c|c|c|c|c|c|}
\hline \multirow[b]{3}{*}{ Moddo } & \multirow[b]{3}{*}{ R } & \multicolumn{8}{|c|}{ Resummen del modelelo } \\
\hline & & \multirow[b]{2}{*}{ Reuadlado } & \multirow[b]{2}{*}{$\begin{array}{l}\text { Reualdato } \\
\text { auglatalo }\end{array}$} & \multirow{2}{*}{ 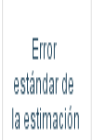 } & \multicolumn{5}{|c|}{ 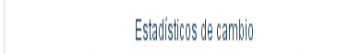 } \\
\hline & & & & & $\begin{array}{l}\text { Cambion en } \\
\text { culadualo }\end{array}$ & Cambionn & gl & 12 & $\begin{array}{c}\text { Sig. Cambli } \\
\text { enf }\end{array}$ \\
\hline 1 & $911^{1}$ & 880 & 818 & 18216 & .830 & 88436 & 1 & 14 & , \\
\hline 2 & $977^{\circ}$ & 955 & 948 &, 33269 &, 125 & 35803 & 1 & 13 & الת \\
\hline \multicolumn{10}{|c|}{ 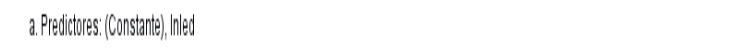 } \\
\hline \multicolumn{10}{|c|}{ 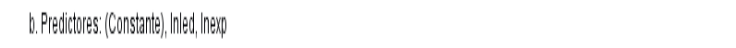 } \\
\hline & depe & diente: $\mid$ PFil & & & & & & & \\
\hline
\end{tabular}

Para el modelo de regresión probado con las dos variables independientes se explica el $94.8 \%$ de la varianza de la variable dependiente. El coeficiente de determinación $\left(\mathrm{r}^{2}\right)$ ajustado es alto lo cual manifiesta que el modelo de regresión lineal múltiple es el adecuado para realizar algún pronostico con errores bajos, en conclusión la ecuación obtenida es la adecuada.

\section{Gráfico 1. Histograma}

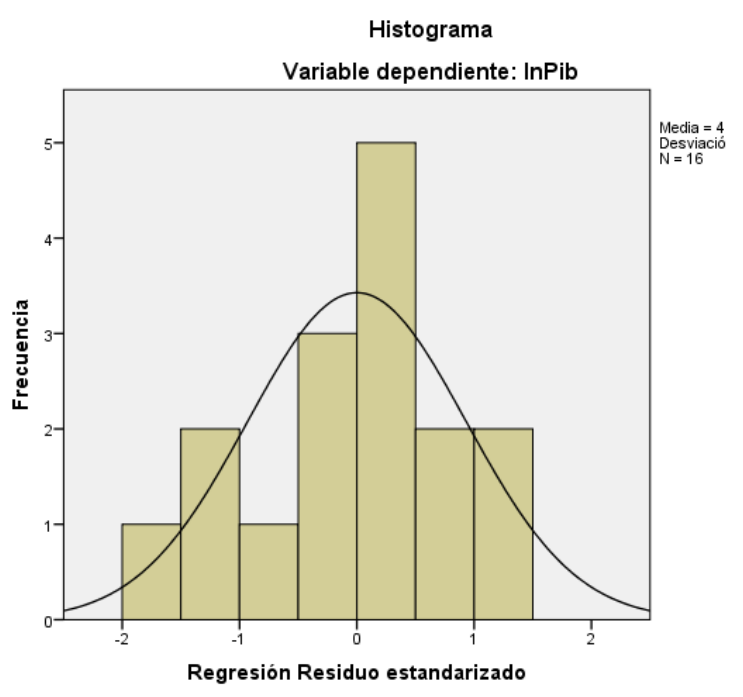

En el gráfico 1 se visualiza al generar una suavización del polígono que se ajusta a una distribución normal.

Con relación al grafico 2 , de normalidad se observa que los puntos están cerca de la línea de tendencia, confirmándose el supuesto de la normalidad
El puntaje obtenido en la prueba de Durbin-Watson de 1.935 indica que hay independencia de errores, ya que está ubicado entre 1.5 y 2.5. Este coeficiente indica que los residuos no están relacionados entre ellos.

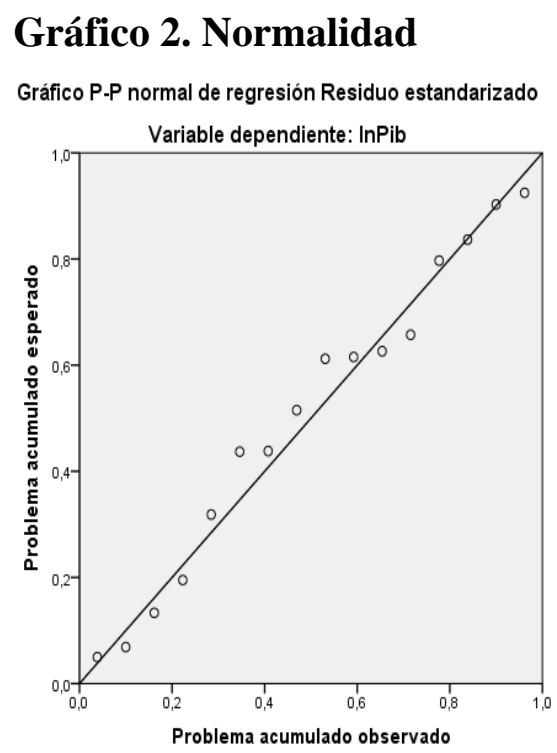

Se concluye, una vez aplicado el método de regresión lineal múltiple y analizados los resultados obtenidos que se cumplen los siguientes supuestos:

a) Linealidad: Relación lineal entre la variable dependiente y cada una de las independientes.

b) Homoscedasticidad: Varianza constante en los $u_{i}$.

c) Independencia de residuos: Covarianza nula entre los $u_{i}$.

d) Normalidad: Los residuos deben estar distribuidos normalmente.

e) No haya multicolinealidad. 


\section{Gráfico 3. Dispersión}

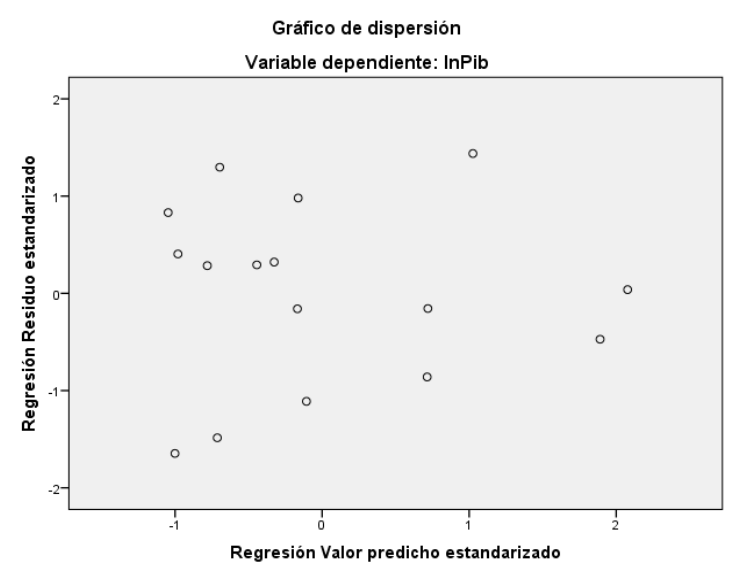

En el gráfico 3 de dispersión de los residuos se observa que hay una compensación referente al cero que permite mantener la relación lineal, confirmándose el supuesto de la homocedasticidad, esto es la varianza de los residuos es constante y no varía en los diferentes niveles del factor. Si el gráfico no presenta patrón alguno, entonces, no se puede rechazar la hipótesis de igualdad de varianzas.

\section{Tabla 5. Prueba ANOVA}

\begin{tabular}{|c|c|c|c|c|c|c|}
\hline \multicolumn{7}{|c|}{ ANOVA $^{a}$} \\
\hline \multicolumn{2}{|c|}{ Modelo } & $\begin{array}{l}\text { Suma de } \\
\text { cuadrados }\end{array}$ & gl & $\begin{array}{c}\text { Media } \\
\text { cuadrática }\end{array}$ & $\mathrm{F}$ & Sig. \\
\hline \multirow[t]{3}{*}{1} & Regresión & 26,405 & 1 & 26,405 & 68,436 &, $000^{b}$ \\
\hline & Residuo & 5,402 & 14 &, 386 & & \\
\hline & Total & 31,807 & 15 & & & \\
\hline \multirow[t]{3}{*}{2} & Regresión & 30,368 & 2 & 15,184 & 137,183 &, $000^{\circ}$ \\
\hline & Residuo & 1,439 & 13 &, 111 & & \\
\hline & Total & 31,807 & 15 & & & \\
\hline \multicolumn{7}{|c|}{ a. Variable dependiente: InPib } \\
\hline \multicolumn{7}{|c|}{ b. Predictores: (Constante), Inled } \\
\hline \multicolumn{7}{|c|}{ c. Predictores: (Constante), Inled, Inexp } \\
\hline
\end{tabular}

Con F igual a 137.18 y un nivel de significancia de 0.000 , se rechaza la hipótesis nula, consecuentemente, el modelo es significativo para la población

\section{CONCLUSIONES}

En este trabajo se analizó el impacto de las exportaciones y de inversión extranjera directa en el crecimiento económico de 16 países latinoamericanos. Las variables están medidas en millones de dólares y en logaritmo natural y se confirma que el MRLM es pertinente para explicar el fenómeno en la muestra analizada que contiene una serie transversal de datos correspondientes a un solo año.

Los resultados sugieren que las exportaciones son un determinante del crecimiento económico, al sector público le corresponde generar externalidades que apoyen a este sector, al existir una alta correlación directa entre exportaciones e importaciones se debe tomar en cuenta que las políticas proteccionistas afectan al comercio exterior de los países.

En el presente estudio se identificó que la IED promueve el crecimiento económico, ya que incorpora recursos frescos que permiten equilibrar la balanza de pagos y motiva la transformación industrial al generar efectos positivos traducidos en elevación de la productividad, del valor agregado y de las exportaciones

$\mathrm{Si}$ bien la variable volumen negociado en el mercado de valores fue descartada en el presente estudio por tener una alta correlación con la IED y por ser la menos correlacionada con la variable dependiente. A los diferentes países les corresponde incentivar a este mercado que constituye para las empresas una importante fuente de financiamiento de recursos a menor costo, mayores volúmenes y plazos.

Las exportaciones y la IED promueven el crecimiento económico en Latinoamérica, siendo un dato importante para los organismos que hacen políticas públicas y es concordante con la mayoría de literatura 
al respecto. Finalmente, se recomienda ampliar la presente investigación, utilizando series de tiempo o datos panel.

\section{REFERENCIAS BIBLIOGRÁFICAS}

Álvarez, A., Barraza, S., \& Legato, A. (2009). "Inversion extranjera directa y crecimiento económico en Latinoamérica". Información Tecnológica, 20 (6): 115-124. 〈doi:10.1612/inf.tecnol.4116it.0 8).

Arbeláez, M. (marzo de 2004). Obtenido de 〈http://www.repository.fedesarro llo.org.co/bitstream/handle/1144 5/1287/Repor_Marzo_2004_Ani f_y_Fedesarrollo.pdf?sequence $=$ 3 \&is Allowed $=\mathrm{y}$.

Blomstrom, M., \& Ye Wang, J. (1989). "Foreign Investment and Technology Transfer: A Simple Model". Economic Review 36: 137-155. 〈doi:10.3386/w2958〉.

Comisión Económica para América Latina y el Caribe (CEPAL). (2004). Desarrollo productivo en economías abiertas. Santiago de Chile: CEPAL.

Comisión Económica para América Latina y el Caribe. (2016). Estudio económico de América Latina y el Caribe. Santiago de Chile: CEPAL. Obtenido de <repositorio.cepal.org/bitstream/ handle/11362/40326/86/S16007 99_es.pdf .

Demirguc-Kunt, A., \& Levine, R. (1993). Stock Market Development and Financial Intermediary Growth : A Research Agenda. Washington:
Policy Research Working Paper Series.

Dunning, J., \& Lundan, S. (2008). Multinational Enterprises and de Global Economy. Massachusetts: Edward Elgar Publishing Limited, Inc.

Dussel, E., \& Galindo, L. (2007). Inversión extranjera directa en México: Desempeño y potencial una perspectiva macro, meso, micro y territorial. México DF: Siglo XXI.

Hicks, J. (1969). A Theory of Economic History, vol. 163. Oxford: Clarendon paperbacks.

Kozikowsky. (2013). Finanzas internacionales. México DF: McGraw-Hill.

Levine, R., \& Zervos, S. (1998). "Stocks Markets, Banks, and Economic Growth". The American Economic Review, 3 (88): 537-558. Obtenido de http://www.jstor.org/stable/116 848?.

Marshall, P., \& Walker, P. (2000). "Day-of-the-Week and Size Effects in Emerging Markets: Evidence from Chile". Revista de Análisis Económico, 15 (3): 89-108. Obtenido de 〈http://www.raeear.org/index.php/rae/article/vie w/105/202>.

Rivas, S., \& Puebla, A. (2016). "Inversión extranjera directa y crecimiento económico". Revista Mexicana de Economía $y$ Finanzas, 11 (2): 51-75. Obtenido de 〈http://www.scielo.org.mx/pdf/r 
mef/v11n2/1665-5346-rmef-1102-00051.pdf .

Rueda, A. (2005). Para entender la bolsa. Financiamiento $e$ inversión en el mercado de valores, $2^{\mathrm{a}}$. ed. México DF: International Thomson Editores.

Smith, A. (1994). Investigación sobre la naturaleza y causas de la riqueza de las naciones. México DF: Fondo de Cultura Económica.

Thirlwall, A. (2003). La naturaleza del crecimiento económico: Un marco alternativo para comprender el desempeño económico de las naciones. México DF: Fondo de Cultura Económica. 